Corrigendum

\title{
Corrigendum to "Effects of therapeutic hypothermia on the gut microbiota and metabolome of infants suffering hypoxic-ischemic encephalopathy at birth" [Int. J. Biochem. Cell Biol. 93 (December) (2017), 110-118]
}

\author{
C. Watkins ${ }^{\mathrm{a}, \mathrm{b}, \mathrm{e}}$, K. Murphy ${ }^{\mathrm{a}}$, S. Yen ${ }^{\mathrm{f}}$, I. Carafa ${ }^{\mathrm{a}, \mathrm{b}, \mathrm{h}}$, E.M. Dempsey ${ }^{\mathrm{c}, \mathrm{d}}$, C.A. O' Shea ${ }^{\mathrm{d}}$, \\ E. Allen-Vercoe ${ }^{\mathrm{f}}$, R.P. Ross ${ }^{\mathrm{a}, \mathrm{b}, \mathrm{g}}$, C. Stanton ${ }^{\mathrm{a}, \mathrm{b}}$, C.A. Ryan ${ }^{\mathrm{c}, \mathrm{d}, *}$
}

${ }^{a}$ Teagasc Food Research Centre, Moorepark, Fermoy, Co. Cork, Ireland

${ }^{\mathrm{b}}$ APC Microbiome Institute, University College Cork, Ireland

${ }^{\mathrm{c}}$ Infant Centre and Department of Paediatric and Child Health, University College Cork, Ireland

${ }^{\mathrm{d}}$ Department of Neonatology, Cork University Maternity Hospital, Ireland

${ }^{\mathrm{e}}$ Department of Microbiology, University College Cork, Ireland

${ }^{\mathrm{f}}$ Department of Molecular and Cellular Biology, University of Guelph, Ontario, Canada

${ }^{g}$ School of Science, Engineering and Food Science, University College Cork, Cork, Ireland

${ }^{\mathrm{h}}$ Department of Food Quality and Nutrition, Research and Innovation Centre-Fondazione Edmund Mach, San Michele all'Adige, Trento, Italy

The authors regret an error in the original heat map for Figure 4. The heat map highlighted an abundance of Bifidobacterium in the HIE treatment group which was incorrect. A new heat map has been generated which agrees with the results in the paper (see corrected Figure 4 below).

There is no correction required in the main text of the manuscript, just a correction for the legend for Fig. 4, as written below.

The authors would like to apologise for any inconvenience caused.

DOI of original article: https://doi.org/10.1016/j.biocel.2017.08.017

* Corresponding author at: Infant Centre and Department of Paediatric and Child Health, University College Cork, Ireland.

E-mail address: tony.ryan@ucc.ie (C.A. Ryan). 


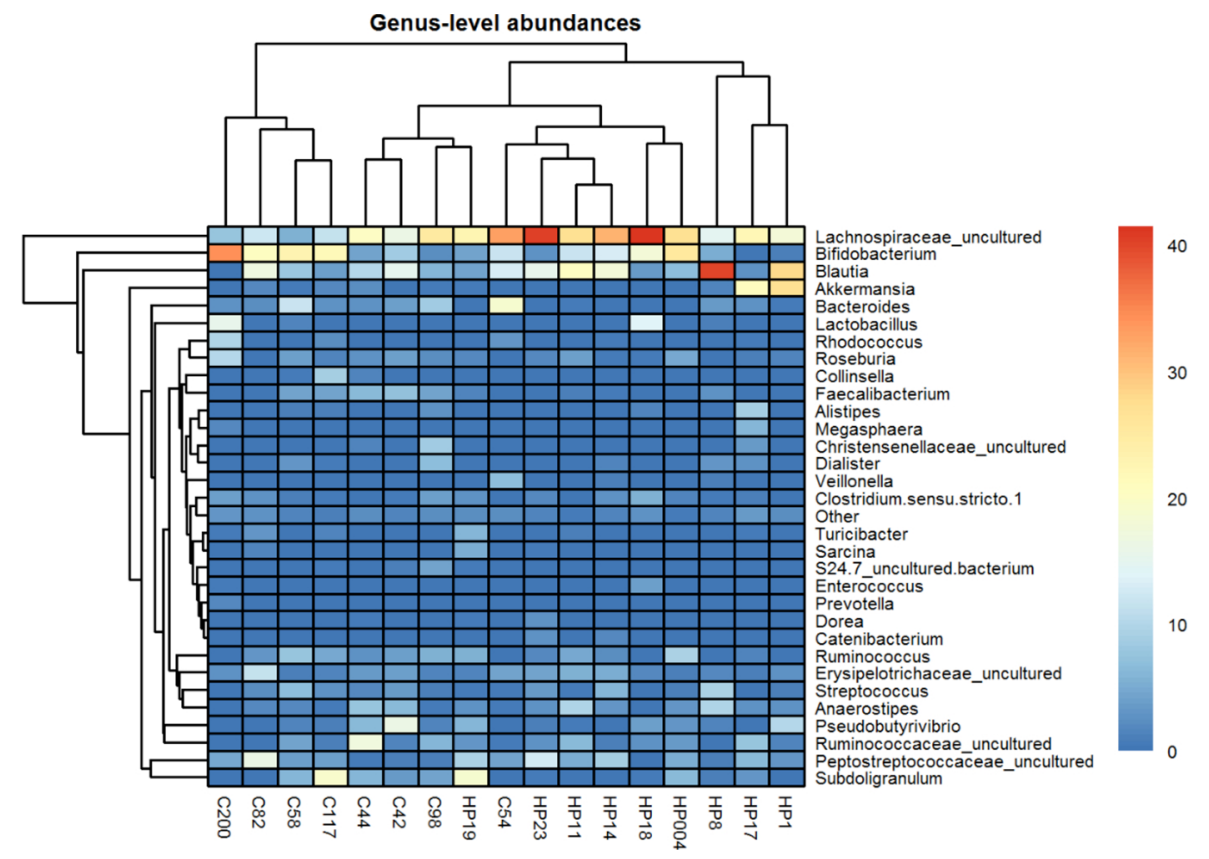

Fig. 4 Hierarchically clustered heat map showing correlations between the relative microbial compositions in each group (control $=\mathrm{C}$, HIE treatment group $=\mathrm{HP}$ ). Clustering was performed with the hclust function in the R package 'pheatmap'. The colour of each tile of the heat map indicates the strength of the correlation for a given genera (red = $35-40 \%$, orange $=\sim 30-35 \%$, yellow $=\sim 20-30 \%$, white $=\sim 15-20 \%$, light blue $=\sim 10-15 \%$, blue $=0-10 \%)$. 